\title{
FORUM
}

\section{Integrated Pest Management: Theoretical Insights from a Threshold Policy}

\author{
Michel I da Silveira Costa ${ }^{1}$, Lucas Del B Faria ${ }^{2 *}$ \\ ${ }^{1}$ Lab Nacional de Computação Científica, Av Getúlio Vargas 333, Quitandinha, 25651-070, Petrópolis, RJ, Brasil; \\ michel@lncc.br \\ ${ }^{2}$ Setor de Ecologia, Depto de Biologia, Univ Federal de Lavras, 37200-000, Lavras, MG, Brasil; lucasdbf@gmail.com \\ *Author for correspondence: (lucasdbf@gmail.com) \\ Edited by Wesley A C Godoy - ESALQ/USP
}

Neotropical Entomology 39(1):001-008 (2010)

\begin{abstract}
An Integrated Pest Management is formulated as a threshold policy. It is shown that when this strategy is applied to a food web consisting of generalist, specialist predators and endemic and pest prey, the dynamics can be stable and useful from the pest control point of view, despite the dynamical complexities inherent to the application of biocontrol only. In addition, pesticide toxicity depends rather on the species intrinsic parameters than on the chemical agent concentration.
\end{abstract}

KEY WORDS: Pest control, virtual equilibrium, pesticide toxicity

Consumer-resource dynamics models include predatorprey, host-parasitoid and herbivore-plant dynamics, which may present a myriad of dynamical behavior as a result of multiplicity of equilibrium states as well as different initial conditions and parameter values (Murdoch et al 2003, Turchin 2003). Therefore, the disturbance of such dynamically complex systems - which have straightforward applications such as biological control - may be severely impaired by these factors.

Amongst other reasons, host-parasitoid and predatorprey population dynamics have been intensively studied because of the importance of parasitoids and predators as biological control agents (Hassell 1978). In an extensive empirical review, Symondson and collegues (2002) found that generalist predators can be effective biological control agents, significantly reducing pest numbers. Approximately $75 \%$ of cases of manipulative field studies showed that generalist predators were capable of effectively suppressing pest populations. However, the introduction of generalist predators as biological control agents raises the unfortunate possibility that nontarget endemic species can be locally suppressed (Simberloff \& Stiling 1998, Boettner et al 2000, Holt \& Hochberg 2001, Lynch et al 2002, Louda et al 2003).

Recently, Cardinale et al (2003) observed that multienemy assemblage can control herbivorous pest (e.g. pea aphid) more effectively than the predicted sum of each enemy separately. In this view, biological control can be analyzed under a multiple predators structure where a generalist predator attacks both endemic and pest prey, while a specialist feeds only on pest prey. In a shared prey and shared predator setup, it was shown that according to the generalist preference, complex behavior may set in leading populations to undergo sharp oscillations nearing low levels, indicating thus a possible extinction of the endemic prey species (Faria et al 2008).

Integrated Pest Management (hereinafter denoted as IPM) (Stern et al 1954) is a pest control strategy that uses an array of additive methods such as biological control cited above and chemical control. This strategy purports to attain a more efficient control on pest outbreaks, minimizing the direct and indirect effects caused by pesticide application. In this light, this work proposes a simultaneous application of the mentioned biocontrol together with pesticide injection, being the latter dictated by a threshold policy.

A threshold policy can be defined in broad terms as follows: control (grazing, harvesting, pesticide application etc.) is suppressed when a specific species abundance is below a previously chosen threshold density; above the threshold, control is applied. It is worthwhile to mention that along with constant harvest rate, fixed proportional harvest and fixed escapement level, threshold policy is an alternative strategy used to exploit (disturb) consumer-resource systems, and its application can be seen in areas such as fishery (Quinn et al 1990, Colie \& Spencer 1993, Quinn \& Deriso 2000), terrestrial harvesting (Jozen et al 2003), grazing (Noy-Meir 1975), conflict uses of aquatic vegetation (Van Nes et al 2002), control of nonnative predators (Sabo 2005), to name a few.

Given the variable structure (Utkin 1978) of the threshold policy (i.e., the alternation between control application and interruption), it is shown that proper combinations of threshold densities and pesticide application intensities based on virtual equilibrium (Costa et al 2000) can lead a variable of interest to a previously chosen level despite the possible dynamical complexities in a specific multi-species consumer- 
resource model used in biological control.

In this case, the ultimate behavior of the system under this strategy consists of the so called sliding motion - a very rapid switching between application and interruption of the control action, which in the present IPM context represents a rapid alternation of pesticide spreading and interruption of pesticide spreading. As will be shown, when this strategy is applied to a food web consisting of generalist, specialist predators and endemic and pest prey, the dynamics can be rather stable and useful from the pest control point of view (i.e., precluding pest outbreaks and endemic prey extinction), despite the dynamical complexities inherent to the application of biocontrol only.

This dynamical outcome, bearing in part on virtual equilibrium, could serve as an instrument for other management purposes, for instance, harvest maximization of renewable resources (Meza et al 2005) and control of microbial populations in a chemostat (Costa \& Meza 2006).

It should be emphasized that this analysis is concerned with illustrating possible outcomes, rather than providing an exhaustive study of conditions required for all possible outcomes. A complete investigation of the full range of biologically plausible parameters over the full range of possible initial conditions in models of three or more species is almost impossible to be carried out (Abrams \& Roth 1994a, b).

The outline of the work is as follows. In section 2 the mathematical structure of the proposed threshold policy is laid out. In section 3 a dynamical food web model used in biocontrol is presented. In section 4 the threshold policy is applied to this model under pest integrated management. In section 5 a summary of the results is presented and discussed.

\section{Mathematical Definition of the Threshold Policy}

A threshold policy (hereinafter called TP) can be defined as the function $\varnothing(\tau)$ such that:

$$
\phi(\tau)=\left\{\begin{array}{lll}
1 & \text { if } & \tau>0 \\
0 & \text { if } & \tau \leq 0
\end{array}\right.
$$

where $\tau$ is the previously chosen threshold.

Given a species $N$ with a particular population dynamics, the proposed TP, $\varnothing$ applied to that species will be given by

$$
\frac{d N}{d t}=f(N)-\phi(\tau) g(N)
$$

where $\varnothing(\tau)$ is defined as in (1), and

$$
\tau=N-N_{t h}
$$

Or equivalently,

$$
\frac{d N}{d t}=\left\{\begin{array}{ccc}
f(N)-g(N) & \text { if } & N>N_{t h} \\
f(N) & \text { if } & N \leq N_{t h}
\end{array}\right.
$$

where $f(N)$ is the species growth rate, $g(N)$ is a density dependent function dictating any species removal rate, and $N_{t h}$ a threshold level. The intensity of the control action depends on whether $N$ is above or below $N_{t h}$. For any finite number of variables involved this policy creates two systems with their own equilibrium points, separated by the threshold level [actually, two structures, and hence the name variable structure system (Utkin 1977, 1978)]. If the equilibrium points are located in their opposite regions, they are named virtual equilibrium points. Otherwise, they are called real equilibrium points (see Fig 1 for a hypothetical case of two variables, $N$ and $P$ ). In case the locally stable equilibrium points are virtual, they will never be attained since the dynamics changes as soon as the trajectories cross the threshold $N_{t h}$. From this setup a sliding mode (Utkin 1992) along $N_{\text {th }}$ may ensue, if in its vicinity the vector fields of both structures are directed toward each other [see Fig 1, although the existence of virtual equilibria is not a necessary condition for the occurrence of a sliding regime (Dercole et al 2003)]. In the present context, this dynamical behavior consists of rapidly alternating pesticide application and suppression.

\section{A Food Web Biocontrol Model}

The structure of the model to be analyzed consists of two non-interacting prey, $N_{p}, N_{e}$, a pest and an endemic prey, respectively, which share a generalist predator, $P_{g}$; in addition there is a specialist predator, $P_{s}$, attacking specifically the pest prey, as shown in Fig 2.

A model for the setting in Fig 2 can have the following form (Faria et al 2008):

$$
\begin{aligned}
& \frac{d N_{p}}{d t}=r_{p} N_{p}\left(1-\frac{N_{p}}{K_{p}}\right)-\frac{a_{s} N_{p}}{1+a_{s} T_{h s} N_{p}} P_{s}- \\
& \frac{(1-p) a_{p} N_{p}}{1+(1-p) a_{p} T_{h p} N_{p}+p a_{e} T_{h e} N_{e}} P_{g}
\end{aligned}
$$

$\frac{d N_{e}}{d t}=r_{e} N_{e}\left(1-\frac{N_{e}}{K_{e}}\right)-\frac{p a_{e} N_{e}}{1+(1-p) a_{p} T_{h p} N_{p}+p a_{e} T_{h e} N_{e}} P_{g}$

$\frac{d P_{g}}{d t}=c_{g}\left(\frac{(1-p) a_{p} N_{p}+p a_{e} N_{e}}{1+(1-p) a_{p} T_{h p} N_{p}+p a_{e} T_{h e} N_{e}}\right) P_{g}-d_{g} P_{g}$

$\frac{d P_{s}}{d t}=c_{s}\left(\frac{a_{s} N_{p}}{1+a_{s} T_{h s} N_{p}}\right) P_{s}-d_{s} P_{s}$

Here, $N_{p}, N_{e}$, represent pest and endemic prey populations, respectively; $P_{g}$, a generalist and $P_{s}$ specialist predator, that attacks specifically $N_{p} . r_{i}$ and $K_{i}(i=p, e)$ are the prey growth rate and carrying capacity; $c_{i}$ is the efficiency conversion of biomass prey $i$; for the generalist predator $a_{i}$ is the attack 


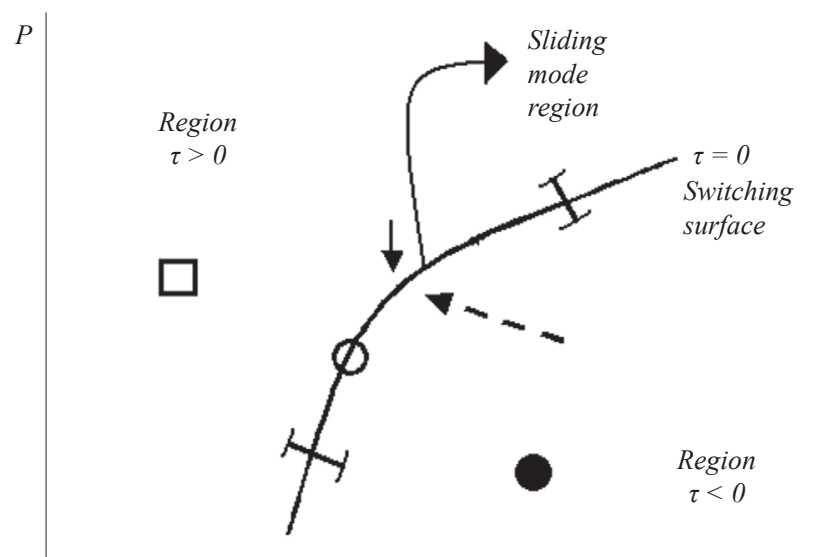

Fig 1 Schematic figure of a sliding mode in a hypothetical phase plane $N \times P$. $\square-$ equilibrium point of $\tau<0 ; \bullet$ - equilibrium point of $\tau>0$. These points are lying in opposite regions, hence they are virtual equilibrium points. $\bigcirc-$ equilibrium point of the sliding regime. Along the sliding mode region the vectors fields of each structure (solid arrow, $\tau>0$, dashed arrow, $\tau<0$ ) are directed toward each other.

rate on prey $i$, and $T_{h \mathrm{i}}$ is its handling time of prey $i ; a_{s}$ is the specialist predator attack rate on the pest prey $\left(N_{p}\right)$ and $T_{h \mathrm{~s}}$ its respective handling time of the pest prey; $d_{i}(j \stackrel{p}{=} g, s)$ is the predator $j$ per capita mortality rate and $p$ is the preference parameter. When $p=0$, the generalist predator diet is entirely directed to the pest $N_{p}$, while $p=1$ denotes a diet composed of endemic prey $N_{e}$ only.

For model (3) a bifurcation diagram for each species as a function of the preference parameter, $p$, shows that for $p=0.65$ (i.e., a diet oriented towards the endemic prey), a complex behavior occurs leading to abrupt oscillations of all components nearing exceedingly low density levels

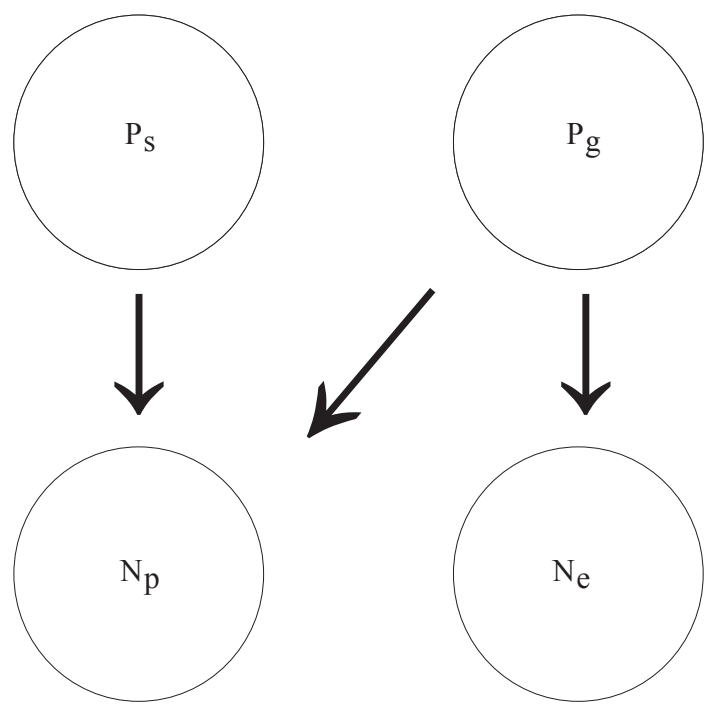

Fig 2 Diagram of predation. $N_{p}$ and $N_{e}$ are a pest and an endemic prey species, respectively; $P_{g}$ is a generalist predator, while $P_{s}$ is a specialist predator preying specifically upon $N_{p}$. Arrows indicate consumption.
(Faria et al 2008). Next, within the context of Integrated Pest Management, a threshold policy regulating pesticide application is put forward with the purpose of suppressing this unstable behavior (due in part to interaction strength dictated by the preference parameter $p$ ), which, among others, may bring about pest outbreaks and endemic prey extinction.

\section{Integrated Pest Management as a Threshold Policy}

Applying the TP (eq. 1) to model (3) yields

$$
\begin{aligned}
& \frac{d N_{p}}{d t}=r_{p} N_{p}\left(1-\frac{N_{p}}{K_{p}}\right)-\frac{a_{s} N_{p}}{1+a_{s} T_{h s} N_{p}} P_{s}- \\
& \frac{(1-p) a_{p} N_{p}}{1+(1-p) a_{p} T_{h p} N_{p}+p a_{e} T_{h e} N_{e}} P_{g}-\varepsilon \phi(\tau) N_{p}
\end{aligned}
$$$$
\frac{d N_{e}}{d t}=r_{e} N_{e}\left(1-\frac{N_{e}}{K_{e}}\right)-
$$$$
\frac{p a_{e} N_{e}}{1+(1-p) a_{p} T_{h p} N_{p}+p a_{e} T_{h e} N_{e}} P_{g}-\varepsilon \phi(\tau) N_{e}
$$

$\frac{d P_{g}}{d t}=c_{g}\left(\frac{(1-p) a_{p} N_{p}+p a_{e} N_{e}}{1+(1-p) a_{p} T_{h p} N_{p}+p a_{e} T_{h e} N_{e}}\right) P_{g}-d_{g} P_{g}-$

$\varepsilon \phi(\tau) P_{g}$

$\frac{d P_{s}}{d t}=c_{s}\left(\frac{a_{s} N_{p}}{1+a_{s} T_{h s} N_{p}}\right) P_{s}-d_{s} P_{s}-\varepsilon \phi(\tau) P_{s}$

with $\varnothing(\tau)$ defined as in (1),

and

$$
\tau=P-P_{\text {sth }},
$$

where $P_{\text {sth }}$ is the specialist predator threshold. $\varepsilon$ is the coefficient that dictates the rate of mortality due to the applied pesticide concentration. Notice that pesticide is supposed to kill all species involved in proportion to their respective densities with the same coefficient (see Davidson et al 2002 for similarly reported cases in pest management). This may represent a severe case, which may very likely be what occurs in practice.

Before proceeding a remark is in order. Model (4) might as well represent an IPM with continuous application of pesticide. To this end one should assume that $\varnothing(\tau) \equiv 1$, and consequently the resulting model would be mathematically equivalent to model (3) (i.e., the variable structure system would disappear). 
System (4) consists of two structures: $(i)$ no pesticide application (for $\tau<0$, i.e., when $P_{s}<P_{\text {sth }}$ ) which generates a complex behavior (Fig 3 a) unstable equilibrium point given by $N_{e}^{*}=0.4, N_{p}^{*}=0.4, P_{g}^{*}=1.6615, P_{s}^{*}=$ 0.49846); $(i i)$ pesticide application with intensity $\varepsilon^{s}>0$ (for $\tau>0$, i.e., when $P_{s}>P_{s t h}$ ) which causes extinction of all species (Fig 3b). These structures correspond to two distinct models which are separated in the phase space $N_{p} \times N_{e} \times P_{g} \times P_{s}$ by the switching plane $P_{s}=P_{s t h}$ (the TP graph). Accordingly, the phase space $N_{p} \times N_{e} \times P_{g} \times P_{s}$ is split into two regions - one for $\mathcal{E}=0$ (below $P_{s t h}{ }^{g}$ ) and one for $\varepsilon>0$ (above $P$, see a schematic view of this variable structure in Fig 1). $P_{s t h}$ was chosen so that the equilibrium points of each structure be virtual (located in opposite regions), hence the stable one (i.e., extinction of all species) can never be attained by its respective dynamics. Besides, the position of the threshold $P_{\text {sth }}$ creates opposed vector fields in some region of its vicinity. This setting engenders a sliding mode (Urtkin 1977, 1978, 1992 ) which corresponds to a rapid alternation of pesticide suppression $(\varepsilon=0)$ and pesticide application $(\varepsilon>0)$ along the specialist threshold $P_{s t h}$. A new steady state situated on the plane $P_{s}=P_{s t h}$ is approached by this dynamics (Fig 3c). In short, given the mathematical structure of the proposed $\mathrm{TP}$, a proper combination of pesticide suppression $(\varepsilon=$ $0)$, pesticide application $(\varepsilon>0)$ and specialist predator thresholds can generate a stabilization process (which prevents pest outbreaks) despite the possibility of complex behavior (attaining quasi-extinction) inherent to model with biocontrol application only (eqs. 3).

To assess how the choice of the specialist threshold $\left(P_{s t h}\right)$ may influence the ultimate behavior of pest species, Fig 4 depicts the long-term dynamics of pest and endemic populations throughout the specialist threshold range 0 $<P_{s t h}<P_{s}^{*}\left(P_{s}^{*}\right.$ is the maximum value of the specialist predator threshold such that the equilibrium points of each structure are virtual). The pest and endemic densities decrease monotonically as $P_{s t h}$ increases, but the latter remains at higher densities than the former.

Being aware of the toxicity incurred from pesticide application, it is noteworthy to assess the rate of pesticide injection when such stable behavior takes place. Since the sliding mode evolves on the threshold $\tau=0$, its dynamics is given by the time derivative of $\tau$, i.e.

$$
\frac{d \tau}{d t}=\frac{d P_{s t h}}{d t}=0
$$

From the equation of $P_{s}$ the equivalent per capita pesticide injection rate $\varnothing_{\text {equiv }}(\tau) \varepsilon$ (Utkin 1992) can be calculated according to

$$
\phi_{\text {equiv }}(\tau) \varepsilon=c_{s}\left(\frac{a_{s} N_{p}}{1+a_{s} T_{h s} N_{p}}\right)-d_{s}
$$

which does not depend on the coefficient that dictates the rate of mortality due to the applied pesticide concentration $\varepsilon$, but rather on the intrinsic parameter values of the species. In other words, toxicity, generally calculated as a function of pesticide injection rate, becomes decoupled from this factor. In addition, the proposed strategy does not interfere in the intensity of pesticide spreading (e.g., by imposing limits on $\varepsilon)$, which, as is commonly known, encounters enforcement difficulties.

At this juncture, it is important to stress that the choice of the threshold and the variables on which the disturbances are applied depends primarily on each specific context. For instance, in IPM a more natural threshold would consist of pest species. However, in that case, simulations evidenced that notwithstanding the pest stabilization around a previously chosen level, both predators tended to extinction - an unacceptable outcome for IPM (not shown here).

\section{Discussion}

Along with other management strategies, threshold policies are undertaken in many fields of renewable resource management such as stock removal in grazing, fisheries, conflicts of aquatic vegetation use as well as in species conservation (Lande et al 1997). In this work an Integrated Pest Management (IPM) - a pest control strategy that uses an array of additive methods such as biological and chemical control - was devised as a threshold policy. Specifically, the IPM problem could be cast as follows, amongst other possibilities.

A preliminary risk/benefit analysis for biological control introductions should take the following points into account:

(1) Avoid the use of exotic generalist predators and parasitoids, which usually feed upon a greater number of non-target species, and induce therefore amplified direct and indirect effects on these species via food webs and cross-linkages (Simberloff \& Stiling 1996, Louda et al 2003)

(2) Expand host-specificity testing for the specialist predator before releasing the biocontrol, including feeding preference tests in choice, no-choice, starvation, functional responses and others. This procedure may avoid specialist predators that attack nontarget species (Louda et al 2003)

Given that the assorted predator species (as a result of the procedure above) correspond to a biocontrol with chaotic dynamics, the proposed IPM can convert this complex behavior into a fairly stable one with coexistence of all species. To achieve such outcome, the elaboration of the IPM followed three basic steps:

(1) Calculate the equilibrium points of each structure (different pesticide application intensities) or their range of values due to parameter uncertainty.

(2) Assign a pesticide application intensity to one of the food web structures so as to eliminate as many equilibrium points of that structure as possible (hence narrowing down the number of potential attractors).

(3) The remaining equilibrium points of each structure (or their respective sets of equilibrium points due to parameter uncertainty) should become virtual by the choice of the specialist predator threshold level. 


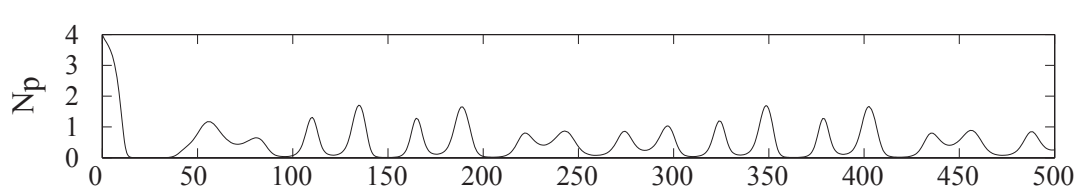

a
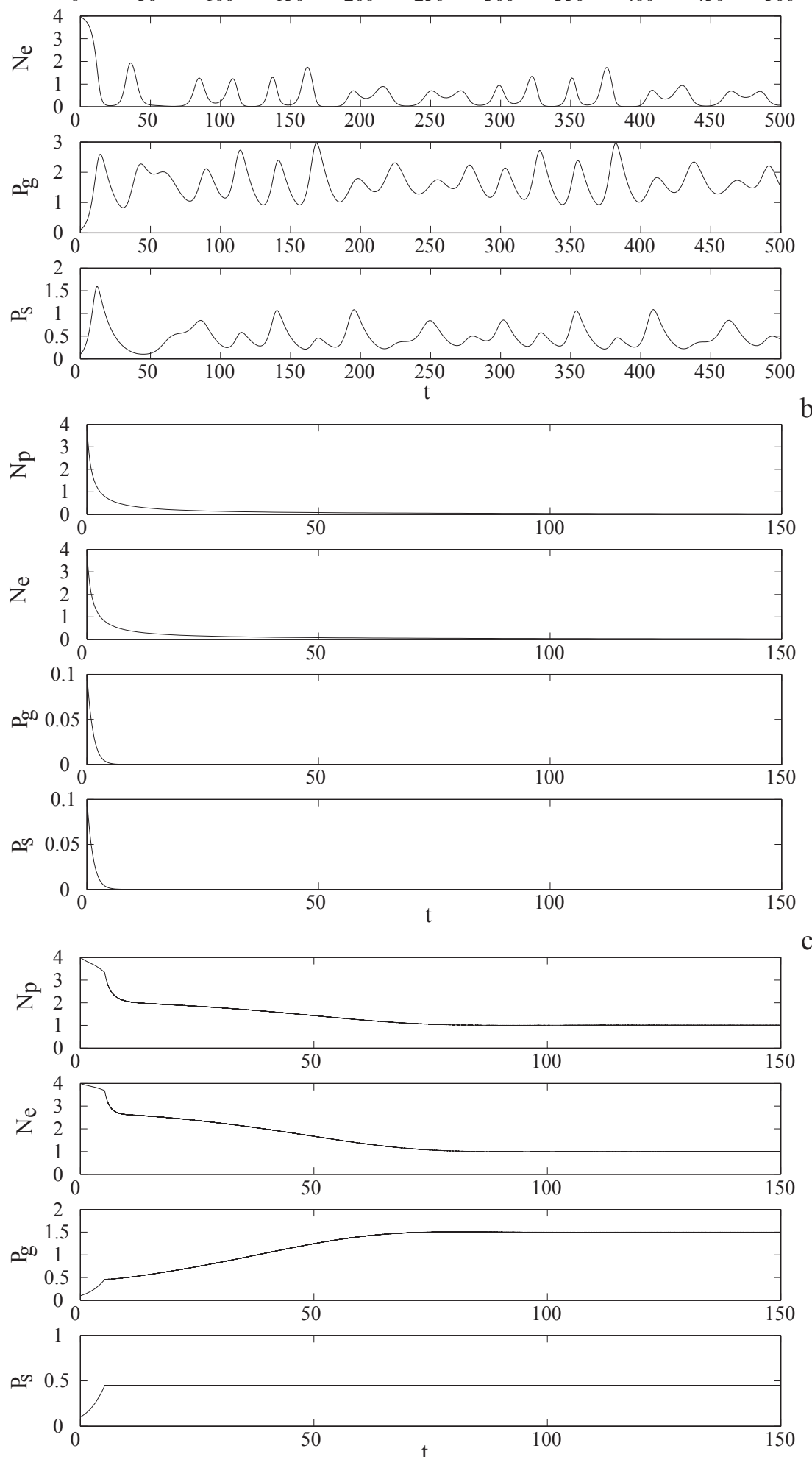

Fig 3 a) Chaotic dynamics for $p=0.65$ and $\varepsilon=0$ (no pesticide application); b) Extinction of all species for $\varepsilon=1$ (application of high pesticide concentration); c) Stabilization (for $p=0.65$ ) by means of a TP in the pesticide control. From top to bottom: $N_{\text {, }}$ $N_{e}, P_{g}$ and $P_{s}$. Note that the specialist predator stabilize in the previously chosen level $P_{\text {sth }}=0.45$. Parameters values: $r_{p}=r_{e}=1 . b^{\prime}$, $K_{p}^{e}={ }^{g} K_{e}=4.0, T_{h p}=T_{h e}=T_{h s}=0.5, a_{p}=a_{e}=a_{s}=1.0, p=0.65, c_{g}=c_{s}=0.3, \varepsilon=1.0, P_{s t h}^{s t h}=0.45$. 

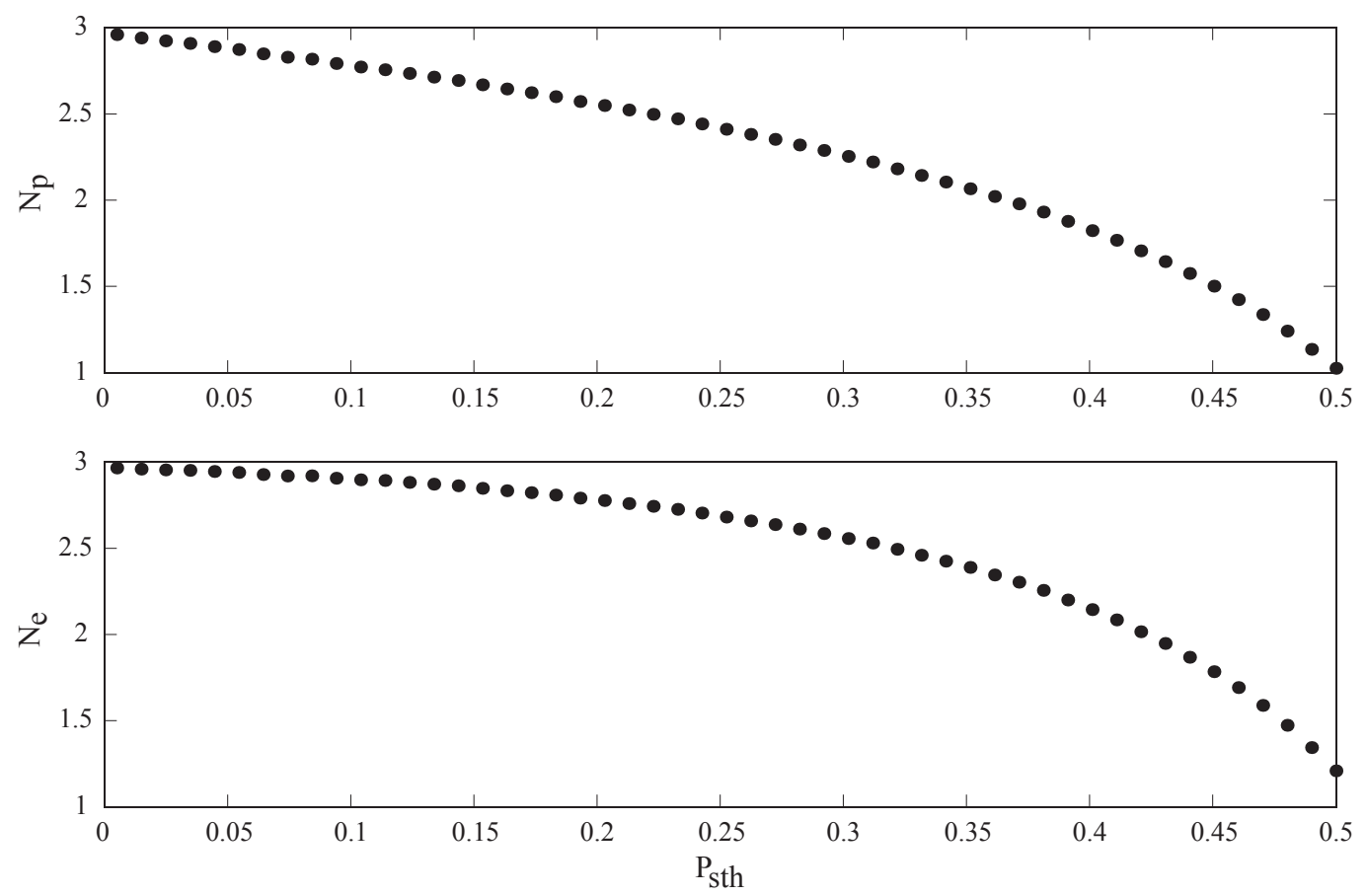

Fig 4 Long term dynamics along values of specialist threshold $P<P_{s t h}<P_{s}^{*}$. Top: pest population $\left(N_{p}\right)$; bottom: endemic prey population $\left(N_{e}\right)$. An increase in a specialist predator threshold, $P_{s t h}{ }^{*}$, incurs in a monotonic decrease in both prey populations.

These guidelines determine the size of the region of the phase space where the policy can be successfully implemented and explain the use of high pesticide concentration (usually causing overall species extinction).

In a food web model with shared predator and prey (eqs. 3 ) the three conditions cited above were fulfilled and the result of this IPM formulated as a threshold management policy consisted of stabilization (i.e., species coexistence without pest outbreaks) of the related system. In other words, it was shown that a specific feature of the mathematical structure of the TP model, namely virtual equilibrium, can play a determinant role in the resulting dynamics of a specific food web under such strategy in the context of Integrated Pest Management. In fact, given a proper combination of pesticide application intensity, generalist predator preference and specialist predator threshold density, a previously chosen specialist predator level was attained by means of a threshold policy applied to the pesticide application intensity.

The ultimate dynamics consisted of rapid alternation between periods of different pesticide intensities. The result of this stabilization was that all species persisted in their respective stable equilibrium levels determined by the sliding mode regime, as opposed to the chaotic behavior (endowed with pest outbreaks and endemic prey extinction) found with biocontrol only. The proposed strategy was actually based upon the creation of virtual equilibrium points and opposed vector fields of each structure along some vicinity of the switching curve (the specialist predator threshold). This stable behavior occurred despite the dynamical complexity of the food web model related to biocontrol only and/or the direct and indirect effects caused by a combined regime of continuous pesticide application and biocontrol.

In case high pesticide levels are not necessarily needed or implemented, the proposed IPM can still be used, however, with more caution (in choosing the threshold) because of the likely existence of locally real equilibrium points (potential domains of attraction), which may lead the system to undesirable states from the IPM standpoint.

As mentioned before, the choice of the threshold and the variables on which the disturbances are applied depends primarily on each specific context. Simulations evidenced that despite the occurrence of pest stabilization around a previously chosen level, both predators tended to extinction - an unacceptable outcome for IPM - when the chosen threshold consisted of the pest species.

It is important to remind that pest prey species eradication is not required, since this outcome would inevitably lead to a generalist predator - endemic prey system that could tend to extinction by means of sharp oscillations - an other unacceptable result from the IPM standpoint. In this case, an occasional immigration of pest prey (not included in the model) could spur a pest outbreak.

Therefore, it is important to pay heed to the fact that the combination of pesticide killing intensity and threshold densities can concur to produce either desirable or undesirable results from the IPM point of view.

Pesticide application and its incurred toxicity inflict detrimental effects both on the environment and on the endemic communities. The number and extent of pesticide applications has reached unprecedented levels and they have been associated with several problems such as habitat loss (e.g. aquatic environment) (Wilcome \& Master 2005), disease emergence (Kiesecker 2002) and declines on nontarget species (Davidson 2002). The IPM suggested in this work partially circumvents this problem, since the rate of pesticide application and its incurred toxicity are dictated 
rather by the intrinsic species parameters than by the term related to pesticide concentration $(\varepsilon)$. Furthermore, it was shown that to generate the stabilization around the specialist threshold $P_{s t h}$, the policy does not require upper limits on the pesticide concentration.

As regards chemical agent spreading, impulsive control strategies are also in order (Lu et al 2004). Basically, they consist of fixed/variable periods of pesticide application, thereby the magnitudes of the pulses (i.e., chemical agent concentration) are evaluated so as to attain pre-specified goals of pest control. By the nature of this policy class, population oscillations commonly ensue (the amplitudes of which may or may not be desirable), besides imposing limits on the pesticide concentration to achieve its purposes - an enforcement prone to encounter difficulties in its practical implementation.

The proposed TP is perhaps best envisaged as another tool to add to the armory of methods: biocontrol, pesticide spreading, simultaneous biocontrol/pesticide spreading, etc., which can all be used to tackle questions of pest control. In this context, the focus of the study concentrated, say, on the "worst" case, that is, the aim was directed to stabilize oscillations (i.e., oscillations of the complete four species food web) with species boom and bust under biocontrol strategy alone (or equivalently, under IPM with continuous injection of pesticide), which occurs, amongst other values, for $p=0.65$. A possible related scenario could be the case where the manager may dispose only of one generalist predator for which $p=$ 0.65 , approximately. Accordingly, from the theoretical point of view, the proposed IPM as a TP proves to be effective as to controlling species dynamics and avoiding species extinction. For other dynamics (e.g., two-point cycle), the proposed IPM could work as well, although biocontrol strategies are also shown to be efficient (Faria et al 2008).

At this juncture, it is worthwhile to stress that the framework presented here concerns a perfect IPM generating an ideal sliding mode. In fact, real systems possess several imperfections such as infrequent and inaccurate measurements of the variables (for instance, see Simberloff \& Stiling 1996) with respect to the problems related to insufficient monitoring of the populations levels in pest management), inherent inertia to measures enforcements (mainly due to high costs related to the rapidly switching pesticide application) which may cause delay or anticipation of the policy application. Consequently, these factors do not enable the occurrence of an ideal instantaneous switching. Nonetheless, if these non idealities are bounded in the vicinity of the threshold and the vector fields of each structure maintained directed towards each other, a non ideal sliding mode still takes place. Accordingly, an ideal sliding behavior should be regarded as a motion limiting process with all non idealities tending to zero (Utkin 1978, 1992). This same procedure can be carried over to a system with random disturbances affecting its dynamics as well as its variables measurement (see Utkin 1992, p.25). With respect to possible applications, given the time scale of the original food web model, these imperfections dictate the time scale of the policy application, which may or may not be suitable in the corresponding real systems.

An explicit account of the imperfections of the proposed
IPM can be performed by couching them into the framework of a hysteresis on-off policy as done in Meza et al (2006). Likewise, relying on virtual equilibria, oscillations within a tube (determined by the bounds inherent to the hysteresis effect) around the specialist predator level $P_{s t h}$ would occur instead of an ideal sliding on the same threshold $P_{s t h}$.

Furthermore, this policy is robust to uncertainties or intrinsic constraints in the model parameters. More specifically, the policy remains effective if the bounds on uncertainties (or constraints) in the parameters can ensure that (a) in some vicinity of the switching curve the vector fields of both structures are opposed; (b) the equilibrium points of each structure stay within a limited region of the phase space in question. The latter limitation may guarantee that they be virtual, a feature on which the proposed TP is partially based.

To sum it up, the approach based on virtual equilibrium may, to some extent, serve as a contribution to the design of more efficient threshold policies for integrated pest management.

\section{References}

Abrams P A, Roth J (1994a) The responses of unstable food chains to enrichment. Evol Ecol 8:150-171.

Abrams P A, Roth J (1994b) The effects of enrichment of threespecies food chains with nonlinear functional response. Ecology 75:1118-1130.

Boettner G H, Elkinton J S, Boettner C J (2000) Effects of a biological control introduction on three nontarget native species of saturniid moths. Cons Biol 14: 1798-1806.

Cardinale B J, Harvey C T, Gross K, Ives A R (2003) Biodiversity and biocontrol: emergent impacts of a multi-enemy assemblage on pest suppression and crop yield in an agroecosystem. Ecol Let 6: 857-865.

Collie J S, Spencer P D (1993) Management strategies for fish populations subject to long term environmental variability and depensatory predation, Tech. Report, 93-02, 629-650 Alaska Sea Grant College.

Costa M I S, Kaszkurewicz E, Bhaya A, Hsu L (2000) Achieving global convergence to an equilibrium population in predatorprey systems by the use of discontinuous harvesting policy Ecol Mod 128: 89-99.

Costa M I S, Meza M E M (2006) Coexistence in a chemostat: Application of a threshold policy. Chem Eng Sci 61: 34003402 .

Davidson C, Shaffer H, Jennings M (2002) Spatial tests of the pesticide drift, habitat destruction, uv-b, and climate-change hypotheses for california amphibian declines. Cons Biol 16: 1588-1601.

Dercole F, Gragnani A, Kuznetsov Y A, Rinaldi S (2003) Numerical Sliding Bifurcation Analysis: An Application to a Relay Control System IEEE Trans. Circuits and Systems I - Fund Theor Appl 50: 1058-1063. 
Faria L D B, Umbanhowar J, McCann K S (2008) The long term and transient implications of multiples predators in biocontrol. Theor Ecol 1: 45-53.

Hassell M P (1978) The dynamics of arthropod predator-prey systems. Princeton, New Jersey.

Holt R D, Hochberg M E (2001) Indirect interactions, community modules and biological control: a theoretical perspective. In: Wajnberg E, Scott J K, Quimby P C (eds.) Evaluating indirect ecological effects of biological control. New York.

Jozen N, Ranta E, Lunberg P, Kaitala V, Linden H (2003) Harvesting Induced Fluctuations? Wild Biol 9: 59-65.

Kiesecker J (2002) Synergism between trematode infection and pesticide exposure: a link to amphibian limb deformities in nature? Proc Nat Acad Sci U.S.A. 99: 9900-9904.

Lande R, Saether B E, Enger S (1997) Threshold harvesting for sustainability of fluctuating resources. Ecology 78: 1341- 1350.

Louda S M, Pemberton R W, Johnson M T, Follett P A (2003) Nontarget effects - The Achilles' Heel of Biological Control? Retrospective analyses to reduce risk associated with biocontrol introductions. Ann Rev Entomol 48: 365-396.

Lu Z, Chi X, Chen L (2004) Impulsive control strategies in biological control of pesticide Theor Pop Biol 64: 39-47.

Lynch L D, Ives A R, Waage J K, Hochberg M E, Thomas M B (2002) The risks of biocontrol: transient impacts and minimum nontarget densities. Ecol Appl 12: 1872-1882.

Meza M E M, Bhaya A, Kaszkurewicz E, Costa M I S (2005) Threshold policies control for predator-prey systems using a control Liapunov function approach Theor Pop Biol 67: 273-284.

Meza M E M, Bhaya A, Kaszkurewicz E, Costa M I S (2006) Onoff policy and hysteresis on-off policy control of the herbivorevegetation dynamics in a semi-arid grazing system. Ecol Eng 28: 114-123.

Murdoch W W, Brigs C J, Nisbet R M (2003) Consumer-Resource dynamics. Princeton University Press, USA.
Noy-Meir I (1975) Stability of grazing systems: an application of predator-prey graphs. J Ecol 63: 459-481.

Quinn T J, Deriso R B (2000) Quantitative fish dynamics. Biological resource management series. Oxford University Press, Oxford.

Quinn T J, Fagen R, Zheng J (1990) Threshold management policies for exploited populations. Can J Fish Aqua Sci 47: 2016-2029.

Sabo J L (2005) Stochasticity, population dynamics and trigger harvest of nonnative predator. Ecology 86: 2239-2243.

Simberloff D, Stiling P (1996) How risky is biological control? Ecology 77: 1965-1974.

Stern V, Smith R, Bosch V D R, Hagen K S (1959) The integrated control concept. Hilgardia 29: 81-101.

Symondson W O C, Suderland K D, Greenstone M H (2002) Can the generalist predators be effective biocontrol agents? Ann Rev Entomol 47: 561-594.

Turchin P (2003) Complex Population Dynamics: a theoretical/ empirical synthesis. Princeton.

Utkin V I (1977) Variable structure systems with sliding modes. IEEE Trans Automat Control 22: 212-222.

Utkin V I (1978) Sliding modes and their applications in variable structure systems. Mir, Moscow.

Utkin V I (1992) Sliding modes in control and optimization. Springer-Verlag, Berlin.

Van Nes E H, Scheffer M, Van den Berg M S, Coops H (2002) Aquatic macrophytes: restore, eradicate or is there a compromise? Aqua Bot 72: 387-403.

Wilcove D S, Master L L (2005) How many endangered species are there in the United States? Front Ecol Environ 3: 414-420.

Received 16/VI/09. Accepted 05/I/10. 